\title{
Desarrollo de un Biosensor Amperométrico en Configuración plana para la Cuantificación de Colesterol
}

\author{
Mónica Hernández, Carlos A. Galán*, Giaan A. Álvarez, M. E. Páez \\ Universidad Autónoma del Estado de Hidalgo. Área Académica de Química. Carretera Pachuca \\ Tulancingo Km 4.5, 42184 Mineral de la Reforma, Hidalgo, México. (e-mail: galanv@uaeh.edu.mx, \\ giaan@uaeh.edu.mx, paezh@uaeh.edu.mx) \\ * Autor a quien debe ser dirigida la correspondencia
}

Recibido Dic. 13, 2010; Aceptado Ene. 28, 2011; Versión Final recibida Abr. 04, 2011

\section{Resumen}

Se presenta el desarrollo de un biosensor amperométrico de colesterol en configuración plana, el cual es fabricado mediante procesos serigráficos compatibles con metodologías de producción automatizadas. El dispositivo incorpora tetracianoquinodimetano, TCNQ, como mediador a fin de reducir el potencial de trabajo y minimizar las interferencias. Presenta un intervalo de respuesta lineal de 2-12 $\mathrm{mM}$ con un límite de detección de $1.56 \mathrm{mM}$, requiriendo un volumen de muestra de solo $7.2 \mu \mathrm{L}$. Sus características lo hacen adecuado para el análisis descentralizado de colesterol en suero sanguíneo y alimentos. La propuesta muestra que sin grandes recursos técnicos ni económicos es posible desarrollar biosensores con características de respuesta competitivas y compatibles con la producción en masa.

\section{Development of an Amperometric Biosensor in Planar Configuration for the Quantification of Cholesterol}

\begin{abstract}
The development of a cholesterol amperometric biosensor in planar configuration is presented. The device is constructed by screen printing processes that are compatible with automated production methods. The electrochemical transducer incorporates tetracyanoquinodimethane, $\mathrm{TCNQ}$, as mediator in order to reduce the work potential and minimize interferences. The biosensor presents an interval of lineal answer of 2-12 mM with a limit of detection of $1.56 \mathrm{mM}$, requiring a volume of sample of only $7.2 \mu \mathrm{L}$. Its characteristics make it adequate for the analysis of cholesterol in blood serum and foods. The designed cholesterol amperometric biosensor demonstrates that without major technical or economic resources it is possible to develop biosensors with response characteristics competitive and compatible with mass production.
\end{abstract}

Keywords: amperometric biosensor, cholesterol, TCNQ, screen printing, mediator 


\section{INTRODUCCIÓN}

El colesterol es un lípido que se encuentra en los tejidos corporales y en el plasma sanguíneo de los vertebrados. En el ser humano, la mayoría del colesterol producido por el hígado se utiliza para formar los ácidos biliares que ayudan a asimilar las grasas ingeridas, volviéndolas solubles en el intestino. Además de ser un precursor de los ácidos biliares y un componente de las membranas celulares, la biosíntesis de diversos esteroides importantes, como hormonas y vitaminas, parten del colesterol (Tudela, 2002). No obstante las importantes funciones del colesterol, cuando se excede su nivel normal en sangre $(3.5-6.5 \mathrm{mM})$, se pueden producir enfermedades cardiacas y renales. Un nivel de colesterol total en sangre superior a $6.5 \mathrm{mM}$, implica un incremento en el riesgo de sufrir un ataque al corazón (Tudela, 2002).

Debido a su importancia clínica, es necesario monitorizar la concentración de colesterol en suero sanguíneo y alimentos a fin de contribuir a mejorar la salud de la población. Actualmente, existen diversos métodos de análisis para la determinación del esteroide; entre las técnicas reportadas destacan la espectrofotometría, cromatografía y electroforesis capilar (Ruizgutierrez y Barrón, 1995; Hoving, 1995; Richmond, 1992: Xu, et al., 2002). No obstante las diversas alternativas, muchas de las técnicas requieren de prolongados tiempo de análisis, uso de grandes cantidades de muestra, alto costo y de personal calificado para su implementación, lo cual las hace inadecuadas para el análisis descentralizado. En este sentido, los biosensores electroquímicos han demostrado ser una alternativa valiosa para superar algunas de estas desventajas sin sacrificar la fiabilidad con respecto a los poderosos métodos instrumentales de análisis (Arya, et al., 2008; Montañez, et al., 2011).

Es interesante advertir que en la mayoría de los reportes la metodología de construcción no es compatible con procesos de producción a bajo costo y miniaturización. Estos desarrollos, no hacen factible el análisis de colesterol por el paciente en el hogar o por el médico en su consultorio a fin de tomar oportunamente medidas que conlleven a un mejor estado de salud. Estimulados por estas limitaciones, recientemente se han reportado algunos trabajos sobre el desarrollo de biosensores de colesterol en configuración plana (Carrara, et al., 2008; Domínguez, et al., 2007; Gilmartin y Hart, 1994). Como consecuencia de la mínima solubilidad del colesterol en agua y baja actividad de las enzimas comercialmente disponibles, dichos trabajos involucran normalmente transductores electroquímicos costosos (platino, oro, nanotubos de carbono, etc.) lo que compromete su viabilidad comercial; actualmente sólo existen pocos desarrollos disponibles en el mercado cuyo desempeño debe ser mejorado (Arya, et al., 2008). En el presente trabajo se reporta el desarrollo de un biosensor electroquímico en configuración plana para la determinación de colesterol libre basado en un transductor de grafito que incorpora tetracianoquinodimetano TCNQ como mediador.

\section{EXPERIMENTACIÓN}

Todos las soluciones fueron preparadas con reactivos comerciales de grado analítico. Colesterol (Research Organics), $\mathrm{KCl}$ (Técnica Química, S.A), $\mathrm{KH}_{2} \mathrm{PO}_{4}$ (Sigma), $\mathrm{K}_{2} \mathrm{HPO}_{4}$ (Sigma), $\mathrm{CH}_{3} \mathrm{CH}_{2} \mathrm{OH}$ (Fluka), Triton X-100 (Sigma), 7,7,8,8-Tetracianoquinodimetano (Aldrich), polvo de grafito 1-2 $\mu \mathrm{m}$ (Aldrich) y colesterol oxidasa de Pseudomonas flourescens $2.4 \mathrm{Ul} / \mathrm{mg}$ (Sigma-Aldrich), Carboximetilcelulosa (Sigma-Aldrich). Todas las soluciones acuosas fueron preparadas con agua desionizada Milli Q (Millipore) $(18 \mathrm{M} \Omega \mathrm{cm}$ ). El colesterol se disuelve con $2 \mathrm{ml}$ de isopropanol y $2 \mathrm{ml}$ de Triton X-100 agitando hasta disolver completamente. Posteriormente se afora a $100 \mathrm{~mL}$ con una solución buffer de fosfatos $0.05 \mathrm{M} / \mathrm{KCl} 0.1 \mathrm{M}$ a pH 7.0 (PBS) formándose una suspensión estable. El coctel enzimático se prepara disolviendo colesterol oxidasa en carboximetilcelulosa (CMC) al $0.25 \%$, disuelta previamente en PBS. La mezcla de grafito con TCNQ se prepara mezclando $1 \mathrm{~g}$ de grafito con $10 \mathrm{~mL}$ de TCNQ $0.1 \mathrm{M}$ en acetonitrilo; después de agitar magnéticamente la mezcla por una hora, el material se filtra y seca a $100^{\circ} \mathrm{C}$ por $1 \mathrm{hr}$.

Los biosensores fueron construidos sobre la base de un transductor electroquímico en configuración plana a tres electrodos, fabricado por serigrafía con un equipo manual de impresión, pantallas de 90 hilos y un rasero de poliuretano (Fig. 1). Para la impresión de los electrodos de 
referencia se utilizó la pasta serigráfica comercial de $\mathrm{Ag} / \mathrm{AgCl}$ Electrodag 7019 (Acheson). Para el electrodo de trabajo y contra electrodo se empleó la pasta de grafito Electrodag PF-407C (Acheson) mezclada con un 5\% de grafito-TCNQ. Como encapsulante se utilizó la pasta dieléctrica fotocurable $451 \mathrm{SS}$ (Acheson). El curado fue realizado a $40{ }^{\circ} \mathrm{C}$ por al menos 30 minutos para las resinas termocurables; el fotocurado se llevó a cabo por 1 hora a luz solar directa. Construido el transductor, se inmoviliza la enzima colocando $5 \mu \mathrm{L}$ de cóctel enzimático sobre la superficie del electrodo de trabajo (0.6 UI). Se deja secar en una estufa incubadora a temperatura de $30^{\circ} \mathrm{C}$ por $30 \mathrm{~min}$. Una vez inmovilizada la enzima, con cinta de doble cara y lámina de acetato se forma un canal de un volumen de $7.2 \mathrm{~mm}^{3}$ (Fig. 1).

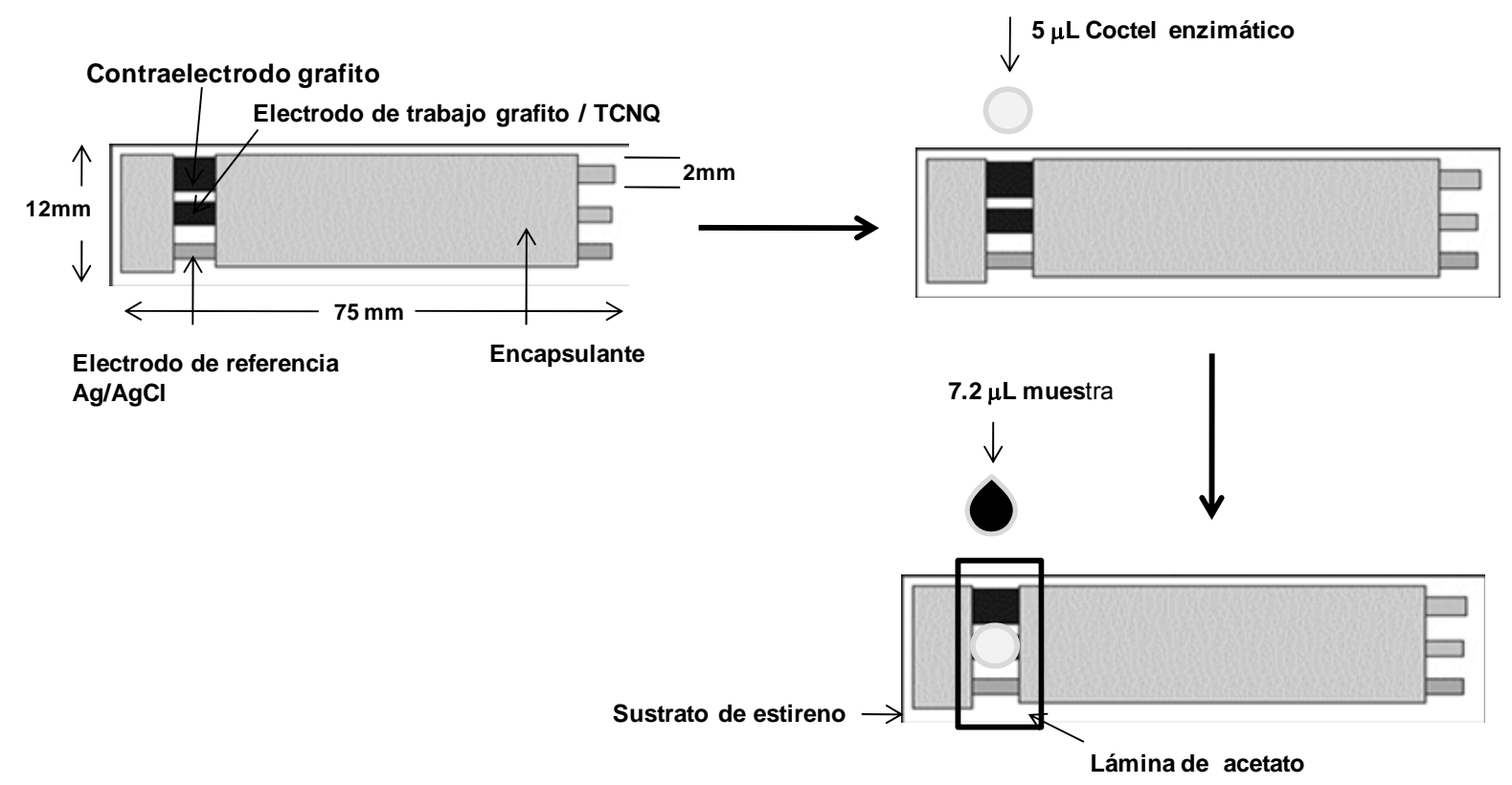

Fig. 1. Biosensores amperométricos de colesterol en configuración plana de tres electrodos.

Los voltamperogramas fueron obtenidos mediante un sistema electroquímico AUTOLAB PGSTAT 30 (Ecochemie). Las determinaciones cronoamperométricas se realizaron mediante un amperímetro LC-4C (BAS). La celda electroquímica se constituyó por un electrodo de referencia de $\mathrm{Ag} / \mathrm{AgCl}(1 \times 2 \mathrm{~mm})$, un electrodo de trabajo de grafito $(2 \times 2 \mathrm{~mm})$ y un contraelectrodo de grafito (4x2mm); todos ellos integrados en un mismo chip (Fig. 1).

\section{RESULTADOS Y DISCUSIÓN}

El funcionamiento convencional de los biosensores de colesterol se basa en la medida de la actividad de la enzima colesterol oxidasa (Ec.1). La enzima colesterol oxidasa (COD) produce coles-4-en-3-ona al entrar en contacto con el colesterol en presencia de oxígeno y agua. El peróxido de hidrógeno producto de la reacción enzimática es oxidado posteriormente cuando se aplica un potencial adecuado sobre el transductor, generando así una corriente eléctrica proporcional a la concentración de colesterol.

$$
\text { Colesterol }+\mathrm{H}_{2} \mathrm{O}+\mathrm{O}_{2} \stackrel{\mathrm{COD}}{\longrightarrow} \text { Coles-4-en-3-ona }+\mathrm{H}_{2} \mathrm{O}_{2}
$$

El peróxido de hidrógeno se oxida a potenciales elevados $(+0.6 \mathrm{~V}$ sobre $\mathrm{Pt},>+1.0 \mathrm{~V}$ sobre grafito respecto al electrodo $\mathrm{Ag} / \mathrm{AgCl}$ ) por lo que especies electroatractivas que se encuentren presentes en las muestras a analizar, como el ácido úrico y ácido ascórbico pueden oxidarse e interferir en el estudio (Shih, 2009). Una forma de evitar estos problemas, es sustituir al oxígeno en su acción regeneradora de la enzima, por un mediador artificial, cuya re-oxidación ocurra a potenciales muy pequeños (delle Noci, et al., 2008) (Ec. 2). 


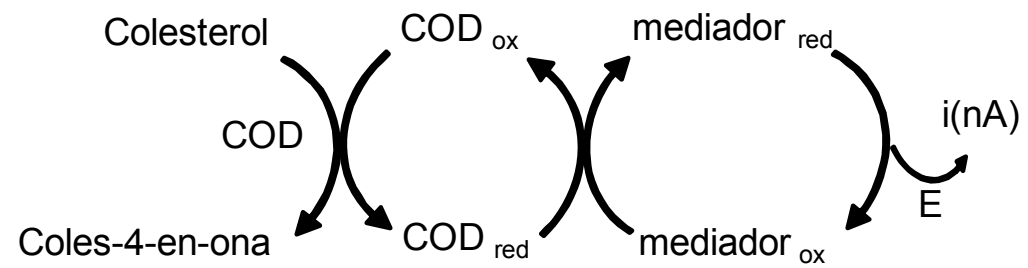

En el desarrollo de biosensores de colesterol basados en COD se han utilizado diversos mediadores (Arya, et al., 2008); sin embargo, hasta el momento no existen reportes en que se use el TCNQ, a pesar de ser considerado un compuesto de baja toxicidad (Kulys, 1992). Con la finalidad de explorar la conveniencia de utilizar tetracianoquinodimetano como mediador, en primera instancia se evaluó el comportamiento del transductor mediante voltametría cíclica (Fig. 2).

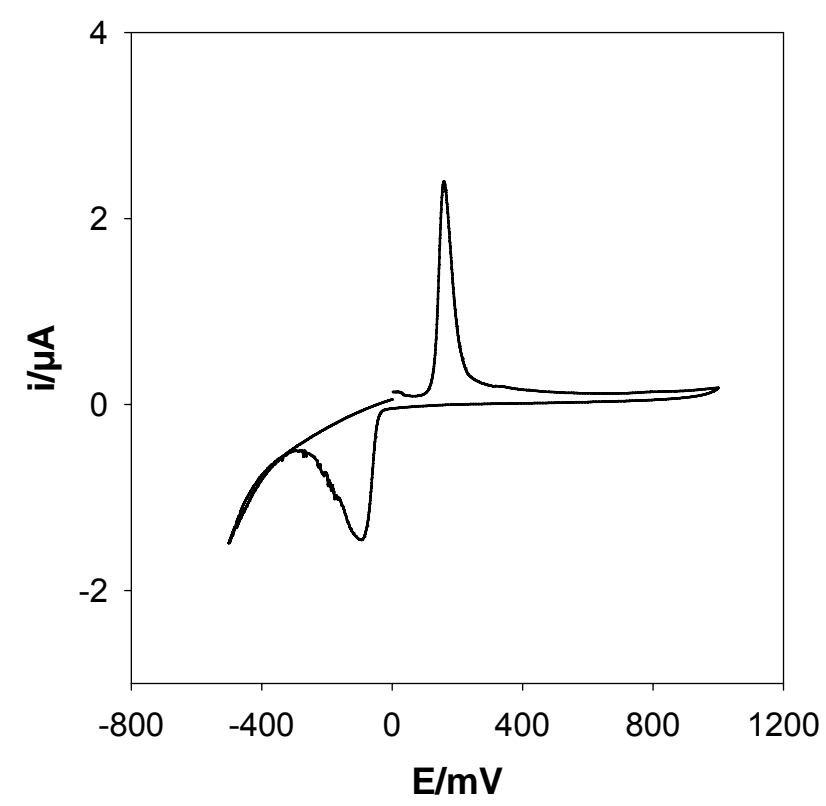

Fig. 2. Voltamperograma cíclico (segundo ciclo) del transductor con $5 \%$ de grafito-TCNQ en solución blanco (PBS) ( $\mathrm{v}=10 \mathrm{mV} / \mathrm{s})$.

Los resultados muestran una señal de oxidación en aproximadamente $150 \mathrm{mV}$ después del primer barrido. Cabe señalar que dicho mediador no presenta originalmente ninguna señal de oxidación ya que se encuentra totalmente oxidado al inicio del experimento; así, al regenerar el sitio activo de la colesterol oxidasa, parte del TCNQ se reducirá, generando una corriente de oxidación al imponer un potencial de trabajo de $150 \mathrm{mV}$ en el biosensor (Ec. 2). Una vez ubicado el potencial de oxidación del TCNQ sobre los transductores construidos, se evaluó la respuesta cronoamperométrica del biosensor de colesterol. Los resultados obtenidos muestran claramente que el biosensor responde al colesterol incrementando la intensidad de corriente respecto al blanco (Fig. 3).

Para obtener los parámetros analíticos del biosensor desarrollado, se realizaron curvas de calibración para colesterol a partir de los cronoamperogramas a los 100 segundos (Fig. 3). Los resultados obtenidos muestran la proporcionalidad de la corriente con respecto a la concentración de colesterol, observando un comportamiento lineal para concentraciones comprendidas en el intervalo 2-12 $\mathrm{mM}$ con un coeficiente de correlación de 0.99. El límite de detección calculado a partir de la curva de calibración conforme al criterio IUPAC (3.3 $\left.\sigma_{b}\right)$ se ubica en $1.56 \mathrm{mM}$ con una pendiente de $221 \mathrm{nA} / \mathrm{mM}$. Es importante destacar que cada uno de los puntos de la curva de calibración se realiza con un biosensor diferentes debido a la naturaleza del dispositivo desechable de un solo uso. Esto implica una buena reproducibilidad de las mediciones ya que la desviación estándar relativa (RSD) se ubica entre $0.08-5.9 \%$ para los triplicados de cada concentración de la curva de calibración. 
Debido a la descomposición de la enzima, el tiempo de vida del dispositivo es de una semana sin refrigerar y un mes en refrigeración $\left(2-8^{\circ} \mathrm{C}\right)$; sin embargo, almacenando al vacío el tiempo de vida se puede incrementar considerablemente.

Conforme al modelo de Michaelis Menten y tomando la intensidad de corriente como la velocidad de la reacción, se encuentra que la $\mathrm{Km}$ aparente es de $0.32 \mathrm{mM}$, ubicándose razonablemente cercana a los valores reportados para la enzima en solución 0.11-0.25 mM y en consecuencia indicando que la actividad de la enzima no ha sido afectada fuertemente por el proceso de inmovilización (Vrielink y Ghisla, 2009).
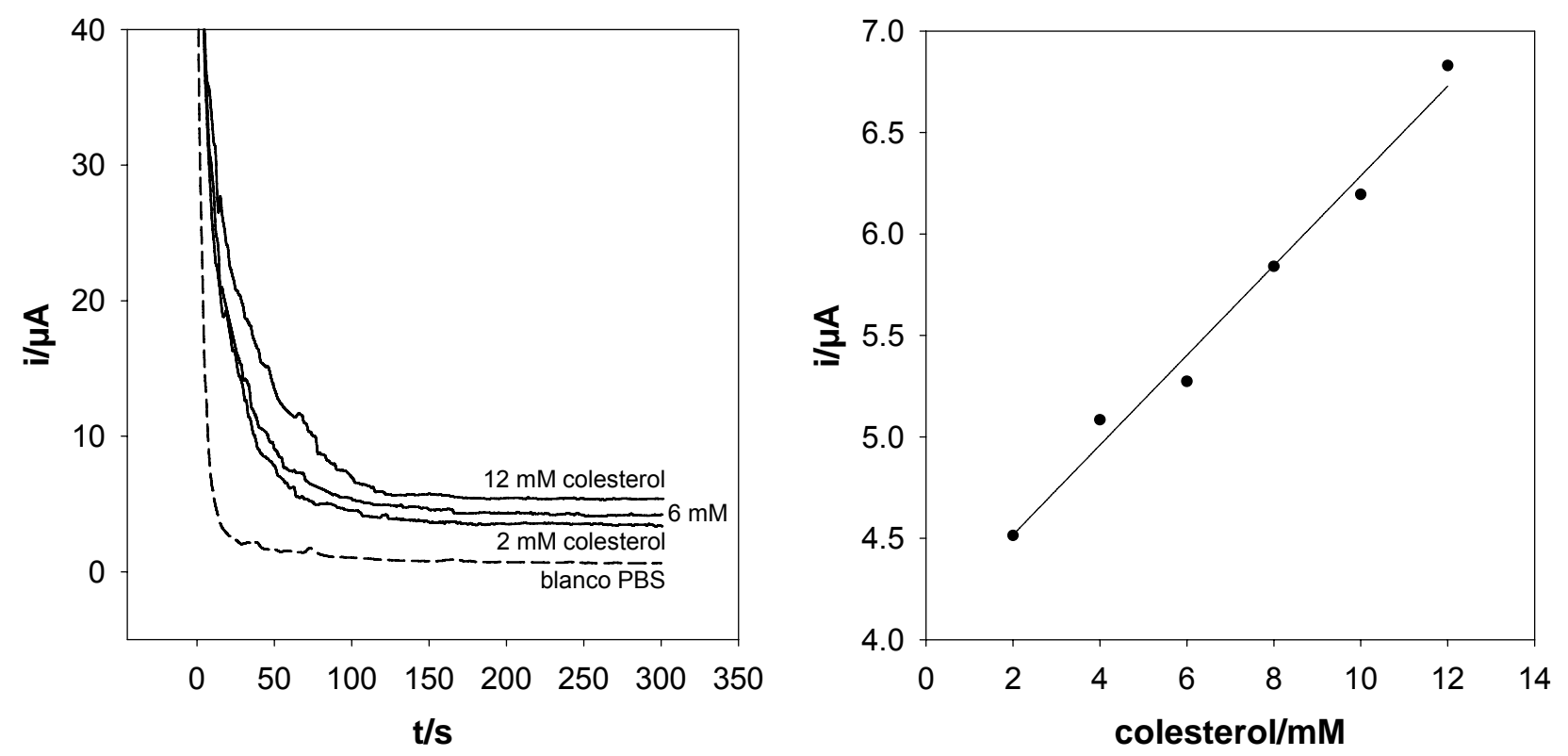

Fig 3. Izquierda: cronoamperometrías de biosensores de colesterol basados en transductores de grafito cargados con TCNQ con membrana de CMC y COD $(E=150 \mathrm{mV})$. Derecha: curva de calibración amperométrica.

La corriente es pequeña para este tipo de biosensores debido a la necesidad de incorporar triton X-100 y propanol para emulsionar el colesterol; asimismo como la escasa actividad de la enzima disponible en el mercado en comparación con la glucosa oxidasa. El dispositivo desarrollado presenta una sensibilidad competitiva, particularmente si se considera que éste no incorpora materiales costosos en su fabricación como nanotubos de carbono, mediadores sofisticados o transductores de metales preciosos (Tabla 1).

Tabla 1. Características de biosensores de colesterol en configuración plana.

\begin{tabular}{|c|c|c|c|}
\hline Transductor & $\begin{array}{l}\text { Mediador o agente } \\
\text { electrocatalítico }\end{array}$ & $\begin{array}{l}\text { Sensibilidad } \\
\left(\mathrm{nA} \mathrm{mM}^{-1} \mathrm{~mm}^{-2}\right)\end{array}$ & Referencias \\
\hline Oro & Sin mediador & 17 & Shen, et al., 2007 \\
\hline Grafito & $\begin{array}{l}\mathrm{Fe}(\mathrm{CN})_{6}{ }^{4-} \text {, nanotubos de } \\
\text { carbono }\end{array}$ & 37 & $\mathrm{Li}$, et al., 2005 \\
\hline Grafito & TCNQ & 55 & Este trabajo \\
\hline Grafito & $\mathrm{Fe}_{3} \mathrm{O}_{4}$ & 87 & Shih, et al., 2009 \\
\hline Grafito & $\begin{array}{l}\text { Grafito, rodio, oro, nanotubos } \\
\text { de carbono, citocromo P450 }\end{array}$ & 1,120 & Carrara, et al., 2008 \\
\hline Grafito & Rodio, citocromo P450 & 6.5 & $\begin{array}{l}\text { Shumyantseva } \\
\text { al., } 2005\end{array}$ \\
\hline
\end{tabular}


Con la finalidad de evaluar la selectividad del dispositivo desarrollado se realizó un estudio de interferencias para ácido ascórbico y dopamina (1.1 mM y $3.7 \mathrm{mM})$, encontrando un incremento menor de la corriente de 3.6 y $1.8 \%$ respectivamente para una concentración de colesterol de 2.5 $\mathrm{mM}$. Debido a que la concentración del ácido ascórbico estudiada supera más de 10 veces el límite normal en sangre y que la concentración de colesterol utilizada en el estudio está por debajo de los niveles normales; es posible afirmar que esta especie no afecta significativamente la respuesta del biosensor. Por otra parte, la dopamina está presente en sangre a niveles traza menores a $0.2 \mu \mathrm{M}$ por lo que su contribución en la corriente del dispositivo desarrollado es despreciable al igual que otras catecolaminas como la epinefrina. Finalmente, aunque al ácido úrico es un conocido interferente cuya concentración en sangre es de aproximadamente 6 veces superior al ácido ascórbico, su potencial de oxidación sobre carbono es superior a $400 \mathrm{mV}$ y en consecuencia no representa una interferencia importante.

\section{CONCLUSIONES}

El biosensor amperométrico de colesterol reportado demuestra que sin grandes recursos técnicos ni económicos es posible desarrollar biosensores con características de respuesta competitivas y compatibles con la producción en masa. El dispositivo de tipo desechable es un prometedor desarrollo para la cuantificación descentralizada de colesterol, ya que el intervalo de concentración encontrado incluye el de interés clínico (3.5-6.5 mM), el volumen requerido para el análisis es mínimo y tiene un costo competitivo de 1.5 dólares americanos que puede ser disminuido significativamente mediante la automatización del proceso de fabricación.

\section{AGRADECIMIENTOS}

El presente trabajo ha sido financiado gracias al apoyo de la Universidad Autónoma del Estado de Hidalgo y del CONACyT (Ref: 62462). MHC agradece la beca CONACyT (203348).

\section{REFERENCIAS}

Arya, S.K., Datta, M., Malhotra, B.D., Recent advances in cholesterol biosensors, Biosensors and Bioelectronics, 23, 1083-1100 (2008).

Carrara, S., Shumyantseva, V.V., Archakov., A. I., Samori, B., Screen-printed electrodes based on carbon nanotubes and cytochrome P450scc for highly sensitive cholesterol biosensors, Biosensors and Bioelectronics, 24(1), 148-150 (2008).

delle Noci, S., Frasconi, M., Favero, G., Tosi, M., Ferri, T., Mazzei, F., Electrochemical kinetic characterization of redox mediated glucose oxidase reactions: A simplified approach, Electroanalysis, 20(2), 163-169 (2008).

Domínguez, R.O., Alonso-Lomillo, M.A., Arcos M. M. J., Recent developments in the field of screen-printed electrodes and their related applications, Talanta, 73, 202-219 (2007).

Gilmartin, M. y Hart, J., Fabrication and characterization of a screen-printed, disposable, amperometric cholesterol biosensor, Analyst, 119(11), 2331-2336 (1994).

Hoving, E. B., Chromatographic methods in the analysis of cholesterol and related lipids, Journal of Chromatography B, 671(1-2), 341-362 (1995).

Kulys, J., Simkeviciene, V., Higgins, I. J., Concerning the toxicity of 2 compounds used as mediators in biosensor devices - 7,7,8,8-tetracyanoquinodimethane (TCNQ) and tetrathiafulvalene (TTF). Biosensors and Bioelectronics, 7, 495-501 (1992).

Li, G., Liao J.M., Hu, G.Q., Ma N.Z. y Wu P.J., Study of carbon nanotube modified biosensor for monitoring total cholesterol in blood, Biosensors and Bioelectronics, 20, 2140-2144 (2005). 
Montañez, J.L., Ramos, E.G., Alegret, S. y Delgado, R.J. Biosensor de glucosa basado en un biocompósito disperso de grafito-epoxi-platino-glucosa oxidasa, Información Tecnológica, 22(1), 29-40 (2011).

Richmond, W., Analytical reviews in clinical biochemistry - The quantitative-analysis of cholesterol, Annals of Clinical Biochemistry, 29, 577-597 (1992).

Ruizgutierrez, V. y Barron, L.J.R., Methods for the analysis of triacylglycerols, Journal of Chromatography B, 671(1-2), 133-168 (1995).

Shen, J. y Liu C. C., Development of a screen-printed cholesterol biosensor: Comparing the performance of gold and platinum as the working electrode material and fabrication using a selfassembly approach, Sensors and Actuators B-Chemical, 120(2), 417-425 (2007).

Shih, W.C., Yang, M.C., Lin, M.S., Development of disposable lipid biosensor for the determination of total cholesterol, Biosensors and Bioelectronics, 24, 1679-1678 (2009).

Shumyantseva V.V. y otros siete autores, C., Direct electron transfer between cytochrome P450scc and gold nanoparticles on screen-printed rhodium-graphite electrodes, Biosensors and Bioelectronics, 21, 217-222 (2005).

Tudela, V., El colesterol: lo bueno y lo malo, $3^{a}$ edición, Fondo de Cultura Económica, Cd de México, México (2002).

Vrielink. A. y Ghisla S., Cholesterol oxidase: biochemistry and structural features, FEBS Journal, 276, 6826-6843 (2009).

Xu X.H., Li R.K., Chen J., Chen P., Ling X.Y., Rao P.F., Quantification of cholesterol in foods using non-aqueous capillary electrophoresis, Journal of Chromatography B, 768(2), 369-373 (2002). 
